Z. Rechtsmediziñ $76,321-333$ (1975)

(C) by Springer-Verlag 1975

\title{
Kehlkopfveränderungen und plötzlicher Tod
}

\author{
W. EISENMENGER, G. BECKMANN und H, -D, TRÖGER \\ Institut für Rechtsmedizin der Ludwig-Maximilians-Universität \\ Müinchen (BRD)
}

Eingegangen am 11. April 1975

Larynx Lesions and Sudden Death

Sumnary: Several recently observed cases of "Sudden Death" caused by larynx lesions give reason for a special discussion of this problem. At the Institute of Legal Medicine only six cases were found in 10.747 autopsies between 1949 and 1970 .

A brief description of the anatomical structure of the larynx is followed by a discussion of the 4 general pathological entities, which might be the etiological cause of laryngeal obstruction:

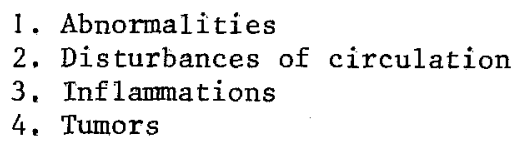

Besides the question of the forensic-medical importance of such fatal cases, the difficulty of evaluating some of the patho-morphologic and histologic findings is discussed. Simultaneous $1 y$ attention is drawn to the frequent 1 ack of a clinical history, so that life-supporting measures such as tracheotomy and others are applied very late or not at all.

Finally the problem of forensic evaluation of larynx-cysts and their etiology is presented. In this context the clarification of a causal relationship with possible previous intubations of the larynx is of major importance.

Zuscomenfassung: Mehrere in letzter Zeit beobachtete Fälle eines "plötzlichen Todes" durch Kehlkopfveränderungen gaben Anlaß zur eingehenden Erörterung dieser Problematik, zumal im Institut für Rechtsmedizin der Universität München unter 10747 Obduktionen in den Jahren 1949 bis 1970 nur 6 Fälle gefunden wurden.

Nach einer kurzen Beschreibung des anatomischen Aufbaues vom Keh1kopf werden entsprechend der in der allgemeinen Pathologie benutzten Gliederung die 4 Faktoren angefthrt, die ätiologisch Ursache eines Kehlkopfverschlusses sein können:
1. Fehlbildungen,
2. Kreislaufstörungen,
3. Entzündungen,
4. Geschwulstbildungen.

Jeder der genannten Faktoren wird gesondert erörtert und die Fälle aus unserem Beobachtungsgut ihnen entsprechend zugeordnet.

Neben der Frage nach der rechtsmedizinischen Bedeutung derartiger Todesfä1le wird die Schwierigkeit der Beurteilung mancher pathomorphologischer und feingeweblicher Befunde diskutiert. Gleichzeitig wird auf die oft leere Anamnese hingewiesen, so daß lebensrettende Maßnahmen, wie zum Beispiel die Notfal1- 
tracheotomie und anderes, gar nicht oder sehr spät einsetzen können. AbschlieBend wird die Problematik der forensischen Begutachtung diskutiert, die sich bei der Abgrenzung der Larynxzysten hinsichtlich ihrer Ätiologie ergibt. Hier ist die Abklärung eines Kausalzusammenhanges zu eventuell vorangegangenen Intubationen von besonderer Bedeutung.

Key words: Keh1kopfveränderungen, plötzlicher Tod - plötzlicher Tod, Keh1kopfveränderungen

Veränderungen im Bereich des Kehlkopfes verursachen nur sehr selten einen plötzlichen Tod aus natürlicher Ursache. In der rechtsmedizinischen Literatur sind die diesbezüglichen Berichte entsprechend selten. So berichtet CAROFF über einen plötzlichen Todesfall, bei dem ein Zungengrundtumor durch einen ventilartigen Verschluß des Kehlkopfes zum Erstickungstod führte. Über einen anderen Fall, bei dem der plötzliche Tod durch ein traumatisch ausgelöstes, angioneurotisches ödem hervorgerufen wurde, berichtet PICK. Die Obduktion ergab hier ein starkes Glottisödem, das asymmetrisch entwickelt war. Der Autor deutete diesen Fall als physikalisch ausgelöstes congenitales angioneurotisches ödem (QUINCKE) und stützte seine Annahme auf eine entsprechende Familienanamnese über 3 Generationen. BOSTRÖM et al. wiesen an Hand von 4 plötzlichen Todesfällen auf das Bild der akuten Epiglottitis hin. Sie beobachteten diese Fälle in einem Zeitraum von 2 Monaten bei 2 Kindern im Alter von 3 und 8 Jahren und 2 Erwachsenen im Alter von 46 und 54 Jahren. In allen 4 Fällen gingen dem Tod nur kurzfristige Beschwerden und Krankheitssymptome von der Art eines a11gemeinen Unwoh1seins voraus.

Am Institut für Rechtsmedizin der Universität München wurden bei 10747 obduktionen in den Jahren 1949 bis 1970 lediglich 6 Fälle von Keh1kopfveränderungen gefunden, die Ursache eines plötzlichen Todes gewesen waren. In jüngster Zeit gelangten kurz aufeinanderfolgend mehrere Todesfä1le zur Beobachtung, bei denen der Tod durch krankhafte Veränderungen im Bereich des Keh1kopfes hervorgerufen wurde. Dies veranlaßte uns, auf die Gesamtproblematik hinzuweisen,

\section{ANATOMISCHE VORBEMERKUNGEN}

Der anatomische Aufbau des Kehlkopfes ist - die einzelnen Bauelemente selbst und ihre Beziehungen zueinander betreffend - darauf ausgerichtet, die spezifische Doppelfunktion des Keh1kopfes zu ermöglichen, nämlich die Stimmbildung und die Pförtnerfunktion für die tiefen Luftwege. Während die Stimmbildung lediglich eine wichtige Funktion in der zwischenmensch1ichen Kommunikation darste11t, ist die Pförtnerfunktion lebensnotwendig. Die topographische Einteilung der vom Kehlkopf umschlossenen Hohlräume orientiert sich am Apparat der Stimmbildung. Man unterscheidet hier 3 Abschnitte: 
Der obere Abschnitt, das Cavum Superius, wird durch den Kehlkopfeingang einschließ1ich des Kehldeckels, die Verbindungsfalten zwischen Kehldeckel und Gießbeckenknorpe1 und Teile des umkleideten Gießbeckenknorpe1s selbst bis zum mittleren Rand des Taschenbandes gebildet. Der untere Abschnitt, das Cavum Inferius, umfaßt den Raum zwischen den Stimmbändern und dem ersten Luftröhrenknorpe1.

Zwischen diesen beiden Regionen liegt das Cavum Intermedium, begrenzt durch die Taschenbänder mit den Morgagnischen Taschen und die Stimmbänder nit den Stimmband taschen.

PLÖTZLICHE TODESFÄLLE AUS NATÜRLICHER URSACHE

Dem plötzlichen Tod aus natürlicher Ursache bei krankhaften Kehlkopfveränderungen liegt ursächlich stets ein Ersticken zugrunde. Der reflektorische Herzstil1stand bei Reizung der Kehlkopfnerven ist zwar ebenfalls eine Möglichkeit des plötzlichen Todes, jedoch wird eine solche Reizung durch natürliche Erkrankungen des Keh1kopfes nie hervorgerufen, sondern meist durch Fremdkörperreize, wie zum Beispiel bei diagnostischen und therapeutischen Eingriffen im Keh1kopfbereich. Ob beim Bolustod, der nicht zum "plötzlichen Tod" im eigentlichen Sinne gerechnet wird, der Tod häufiger durch Ersticken oder durch reflektorischen Herzstillstand hervorgerufen wird, ist noch weitgehend ungeklärt.

Entsprechend dem feingeweblichen Aufbatu und der Topographie sind die zum Tode führenden Kehlkopfveränderungen zumeist im Cavum Superius lokalisiert. Bei der Obduktion ist deshalb das Hauptaugenmerk speziell dieser Region zuzuwenden. Im subglottischen Raum sind dagegen äußerst selten tödliche krankhafte Veränderungen anzutreffen. Bei Krankheitsprozessen im Cavum Intermedium ist in aller Regel mit Störungen der Stimmbildung zu rechnen, die damit frühzeitig Anlaß zu einer ärztlichen Konsultation sind. Ein "plötzlicher Tod" bei Veränderungen in dieser Region wird recht selten beobachtet.

Entsprechend den Grundsätzen der allgemeinen Pathologie kommen als Ursache tödlicher Kehlkopfveränderungen vier ätiologische Faktoren in Frage:

$$
\begin{aligned}
& \text { 1. Feh1bi1dungen } \\
& \text { 2. Kreislaufstörungen } \\
& \text { 3. Entzündungen } \\
& \text { 4. Geschwulstbildungen }
\end{aligned}
$$

Jeder der genannten Faktoren kann für sich ạls Todesursache in. Anspruch genommen werden. Es gibt jedoch auch Kombinationsmöglichkeiten, die wir bei unserem Beobachtungsgut antrafen. 


\section{FEHLBILDUNGEN}

Unter den Fehlbildungen im Kehlkopfbereich, die den Kehlkopfeingang versch1ieBen und zum Erstickungstod führen können, sind die Laryngozelen und die großen Larynxzysten zu nennen. - Die Laryngozelen sind Ausstülpungen der Morgagnischen Ventrikel und ihrer Appendices. Von Bedeutung sind lediglich die inneren Laryngozelen, die die Taschenbänder hochdrängen und das Cavum Intermedium verschlieBen können, Diese Hernien der. Kehlkopfschleimhaut können, insbesondere wenn sie nach außen durch die Membrana hyothyreoidea treten, die Größe einer Kinderfaust erreichen (MATZKER), Histologisch entspricht der Aufbau dem der Morgagnischen Ventrikel mit einem typischen Flimmerepithel und eingestreutem lymphatischem Gewebe, Die Gefährlichkeit der Laryngozelen ist darin zu sehen, daß auslösende Faktoren wie husten, pressen, spielen von Blasinstrumenten sie plötzlich hervortreten lassen und daraus ohne vorherige Beschwerden ein p1ötzlicher Kehlkopfverschluß resultieren kann.

Von den Laryngozelen sind die Larynxzysten abzugrenzen, die uberwiegend am Keh1kopfeingang, fast nie im subglottischen Raum gefunden werden. Unter den, Zysten ist nur ein kleinerer Teil den echten Fehlbildungen zuzurechnen, weitaus häufiger werden Retentionszysten nach Stenosen der Ausführungsgänge von Schleimdrüsen, aber auch Lymphzysten beobachtet. Die Unterscheidung zwischen echten angeborenen zysten und erworbenen Retentionşysten gelingt nicht immer; die feingeweblichen Kriterien, insbesondere das auskleidende Epithe1, sind nicht immer so charakteristisch, daß̣ ein endgültiges Urteil möglich ist, DộRING gibt an, daß in der Literatur bisher nur 29 Fälle kongenitaler Larynxzysten beschrie-ben seien, die bereits unmittelbar nach der Geburt oder nach einigen Lebenstagen obstruktive symptome verursachten. Der von ihm beobachtete Fall eines Neugeborenen, welches 40 Minuten nach der Entbindung tot in eine Klinik eingeliefert wurde, ist inșofern ungewöhnlich, als es sich um eine subglottisehe Zyste handelte und nach dem auskleidenden Epithel von ihm als Retentionszyste eingestuft wurde. Von besonderer Bedeutung ist in diesem Zusammenhang eine Veröffentlichung von WIGGER und TANG, in der eine das Kehlkopflumen verschließende subglottische Zyste beschrieben wird und ein kausaler zusammenhang mit einer länger bestehenden endotrachealen Intubation gefolgert wurde.

Aus rechtsmedizinischer Sicht ist die Tatsache ungewöhnlich, daß Laryngozelen und Larynxzysten für einen "plötzlichen Tod" ursächlich in Anspruch genommen werden, obwohl kaum innerhalb eines kurzen Zeitintervalls eine Vergrößerung der Zysten bis zur Lumenverlegung anzunehmen ist und darüberhinaus anamestische Erhebungen keine Hinweise auf vorangegangene Beschwerden erbringen. 


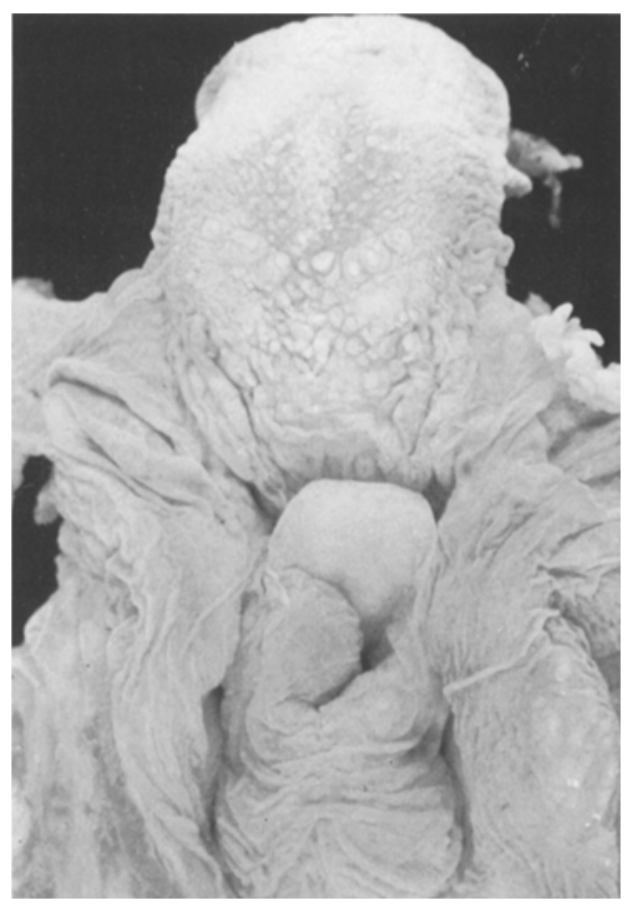

Abb, 1. Verschluß des Kehlkopfeinganges durch 1aterale Kehlkopfzyste

\section{Fal2 1:}

Nach einem Gaststättenbesuch wurde eìn 71-jähriger Mann von seiner Ehefrau im Treppenhaus tot aufgefunden. Außer allgemeinen Kreislaufstörungen waren keine ernsthaften Vorerkrankungen bekannt.

Obduktionsbefunde (GS 108/73):

Walnußgroße, den Keh1kopfeingang vollständig verlegende Zystenbildung an der aryepiglottischen Falte links, mittelgradiges Lungenödem, akute passive Blutüberfiillung der inneren Organe, hochgradige Adipositas, beginnende Stauungszirrhose der Leber, beginnende Altersatrophie des Gehirns, mittelgradige allgemeine Arteriosklerose.

Todesursache:

Ersticken infolge Verschlusses des Kehlkopfeinganges durch eine walnußgroße Zyste (Abb. 1)

Feingeweblicher Befund:

Auskleidung der inneren zystenwandung mit einem mehrzeiligen hochprismatischen Epithel. Bei stärkster Vergrößerung stellenweise ein F1immerhaarbesatz angedeutet erkennbar. Subepithelial eine gut vaskularisierte kernarme Bindegewebs schicht: mit Strängen quergestreifter Muskulatur und Schleimdrüsen sowie Schleimdrïsenausführungsgängen. An einer Stelle ein großkalibriger Ausführungsgang mit einer epithelialen Auskleidung, die in ihrem Aufbau der Innenauskleidung der Zystenwand entspricht (Abb. 2). Im Bindegewebe teils diffus, teils zu Knötchen angeordnete Lymphozyten. Keine Anzeichen von Malignîtät. 


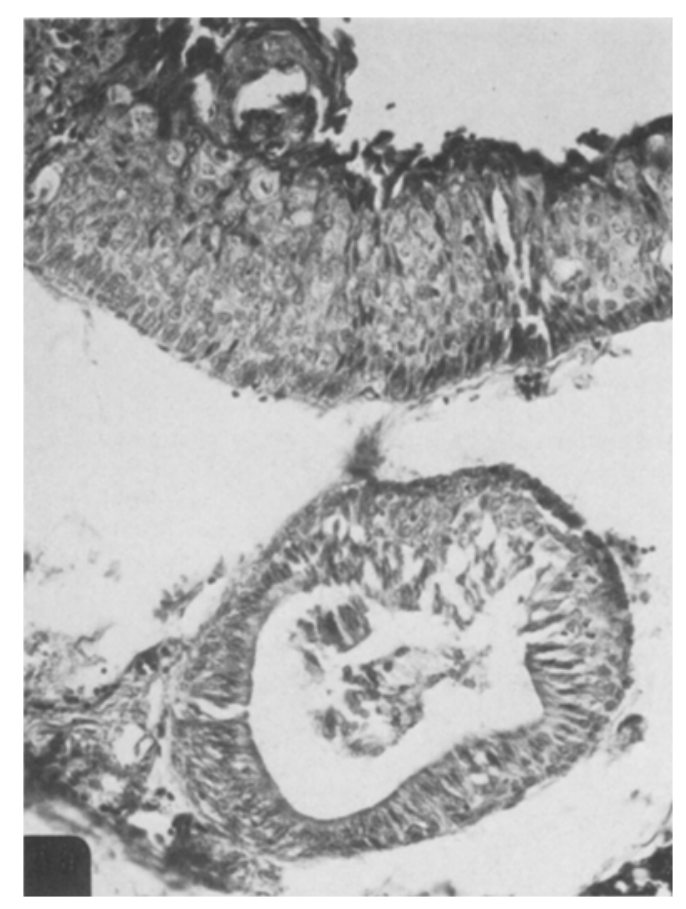

Abb. 2. Ausschnitt aus der Zystenwand: Innenwand der Zyste und Drüsenausführungsgang

\section{KRETSLAUFSTÖRUNGEN}

Eine nicht allzu seltene Todesursache ist der Verschluß des Keh1kopfeinganges durch eine ödematöse Schwellung der Weichteile. Dieses fälschlicherweise als Glottisödem beschriebene Krankheitsbild wird durch eine seröse Infiltration des submucösen Bindebewebes, insbesondere im Bereich der Epiglottis und der aryepiglottischen Falten hervorgerufen. Ein zum Tod führendes ödem durch allgemeine Kreislaufstörungen, zum Beispiel bei Herz- und Nierenkrankheiten, ist extrem selten, klinische Beschwerden dagegen werden in solchen Fällen häufig beobachtet. Das klassische Beispiel eines Kehlkopfödems mit tödlichem Ausgang ist das Quincke-ödem als Folge vasoneurotisch-allergischer Vorgänge. KELLER und WISKOTT ordnen die urticariellen Reaktionen von Haut und Schleimhaut, die Nahrungsmittelallergien und das Quincke-ödem dem Fruhreaktionstyp der allergischen Reaktionen zu. In einer Reihe von Fällen 1äßt sich ein klarer Zusammenhang mit einem bestimmten Allergen nachweisen, jedoch ist verschiedentlich eine Abklärung, ob es sich um eine Nahrungsmitte1- oder Arzneimittelallergie handelt, nicht möglich. Die von PICK in seiner vorerwähnten Arbeit diskutierte erbliche 
Praedisposition zu überschießenden urtikariellen Reaktionen auf physikalische Reize sollte mehr beachtet werden und erscheint uns noch nicht ausreichend abgeklärt. Neben den vasoneurotisch-allergischen ödemen sind ödeme bei Insektenstichen, Verätzungen, Verbrühungen, Operationen und Bestrahlungen im Keh1kopfbereich häufiger anzutreffen. Aus forensischer Sicht können sie jedoch meist nicht der Gruppe des "plötzlichen Todes" zugeordnet werden.

FaZ2 2;

Nach einem Besuch bei seiner geschiedenen Ehefrau wurde ein 25-jähriger Mann in seiner Wohnung tot aufgefunden. Während des Besuches hat te er unter anderem Muscheln gegessen und kurz darauf über Halsbeschwerden geklagt. Diese Beschwerden veranlaßten ihn zur Einnahme eines Antibiotikums, dessen Bezeichnung nicht mehr feststellbar war. Anamnestisch war lediglich zu erfahren, daß er häufiger Entzündungen der Gaumenmandeln mit Antibiotika behandelt hatte.

Obduktionsbefund (GS 64/74):

Höchstgradiges ödem der Schleimhaut im Kehlkopfeingang mit fast völligem Verschluß, Blutfülle der inneren Organe, feinste punktförmige Blutaustritte in den Augenbindehäuten, Volumenvermehrung des Gehirns mit Zeichen von Hirndruck, geringes Lungenödem, flüssiges Leichenblut.

Todesursache: Ersticken bei vermutlich allergischem Kehlkopfödem.

Fal2 3:

Eine 34-jährige Frau bekam seit mehreren Jahren in Abständen von ca. 6 Wochen und 1änger umschriebene Schwellungen am ganzen Körper, hauptsächlich im Gesicht, am Hals und an den Händen. Die Schwellungen traten insbesondere bei psychischer Belastung auf. Am Nachmittag ihres Todestages hatte sie Halsbeschwerden in Form eines Druckgefühls bekommen. Nach dem Abendessen nahm sie wegen der anhaltenden Beschwerden ein vom Hausarzt verordnetes homöopathisches Medikament ein. Gegen 21.45 Uhr verfärbten sich ihre Lippen blau, sie war nicht mehr ansprechbar und verlor das Bewußtsein. Der sofort herbeigerufene Notarzt konnte nur noch den Tod festste1len.

Obduktionsbefund (GS 727/74):

Hochgradige ödematöse Schwellung und sulzig-glasige Durchtränkung der Halsweichteile, verschließendes ödem der Schleimhaut des Kehldeckels und Kehlkopfeinganges, Lungenödem, Hirnödem, akute passive Hyperämie der Organe, punktförmige Blutungen in der Nierenbeckenschleimhaut, chronische Tonsillitis, erbsgroßer AbszeB neben der Iinken Mamille.

Todesursache: Ersticken bei Kehlkopfödem.

\section{ENTZÜNDUNGEN}

Die Todesursache bei Entzündungen im Kehlkopfbereich ist ebenfa11s in einem Ödem der Schleimhäute zu sehen, wobei hier allerdings das histologische Bild zumeist charakteristische zellige Infiltrate aufweist. Die akuten Entzündungen stehen oft in Verbindung mit einer zusätzlichen Entzündurg der oberen und unteren Luftwege durch Bakterien oder Viren. In der eingangs erwähnten Arbeit von BOSTRöM wurden bei 3 Fällen einer tödlichen Epiglottitis Hämophilus influenzae 
nachgewiesen. Diphtherische Entzündungen und spezifische Entzündungen des Keh1kopfes werden heute kaum mehr beobachtet, zumindest nicht als Ursache eines plötzliches Todes. Auf den Kehlkopf lokalisierte Entzündungen in Art einer Phlegmone oder eines Abzesses sind zumeist Folge oberflächlicher Schleimhautläsionen mit bakterieller Besiedelung. Diese lokalen Schleimhautläsionen können jedoch wegen ihrer of nur geringen Ausdehnung häufig postmortal nicht nachgewiesen werden.

FaI2 4:

Ein 5-jähriger Junge erkrankte an einer fieberhaften Erkältung. Nachdem die medikamentöse Behandlung durch den Hausarzt keine Besserung erbrachte, erfolgte die Krankenhausaufnahme. Bei der Aufnahme litt das Kind an einer starken Dyspnoe und war zyanotisch. Es nahm oral noch Medikamente auf, hatte aber nach einer Soludecortin-Injektion einen plötzlichen Atemstillstand. Eine Intubation gelang unter Schwierigkeiten, jedoch blieb die Reanimation erfolglos.

\section{Obduktionsbefund (GS 840/74):}

Verschluß des Keh1kopfeingangs durch ödematöse Schwellung der Schleimhäute, haemorrhagische Tracheobronchitits, Vergrößerung der Halslymphknoten, massiver Hirndruck, fragliche haemorrhagische Lungenentzündung rechts, feste MilzschweIlung.

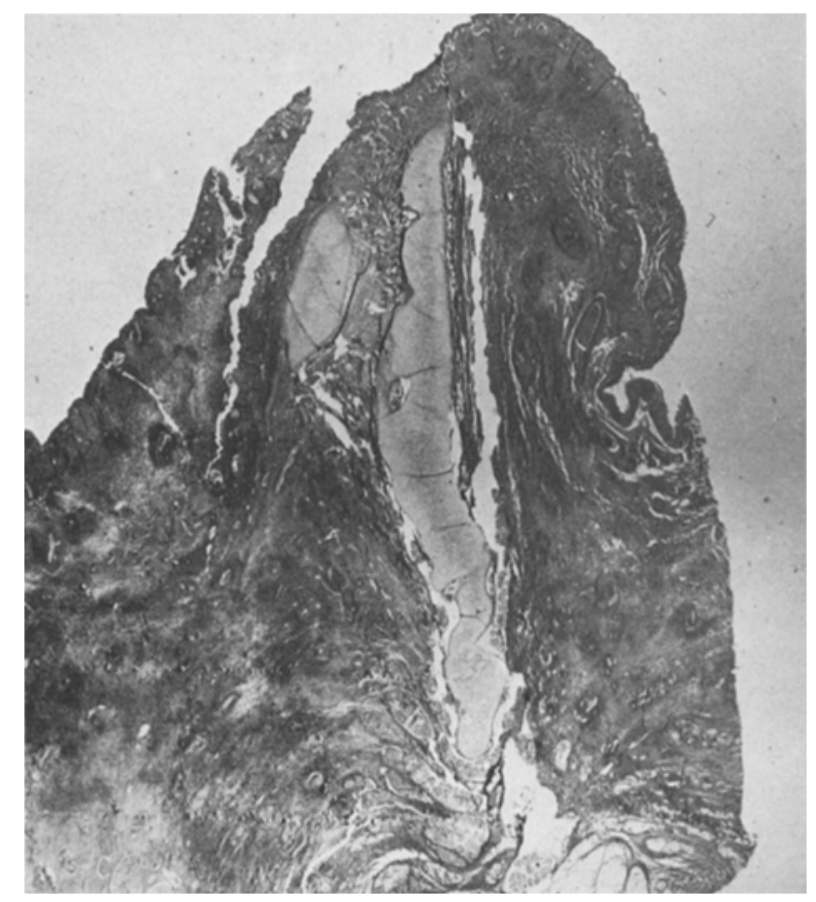

Abb. 3a. Längsschnitt durch die Epiglottis, Entzïndliche Infiltrate in der verdickten Submucosa 


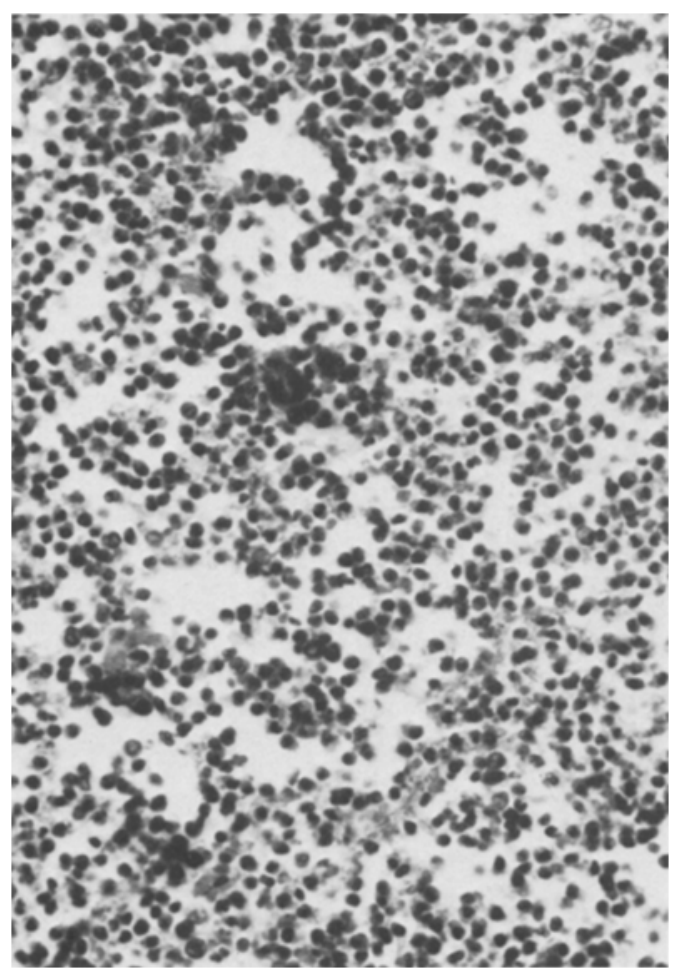

Abb. 3b. Entzündiche Infiltrate in Ausschnittsvergrößerung

Todesursache: Ersticken bei VerschIuß des Kehlkopfeinganges durch ödematöse Schwellung der Schleimhäute.

Histologisch fand sich eine massive leukozytäre Infiltration der gesamten Schleimhaut (Abb. 3).

Bakteriologisch wurden Haemophilus influenzae, Pneumokokken, Escherichia coli und Keime der Klebsiella-Enterobacter-Gruppe nachgewiesen.

\section{EaZ2 5:}

Eine Zeugin bemerkte einen 21-jährigen Mann, der mit ausgebreiteten Armen stark röchelnd auf sie zutaumelte. Gleichzeitig litt er unter Atemnot, Gesicht und Hände waren blau verfärbt. Beim Eintreffen des Notarztes konnte nur noch der Tod festgestellt werden. In der dem Tod vorangegangenen Nacht hatte der Verstorbene im Freundeskreis noch ausgiebig gefeiert.

Obduktionsbefund (GS 735/74):

Abszeßbildung an der Kehlkopfriickwand zwischen Gießbeckenknorpel und Ringknorpel, hochgradiges Schleimhautödem von Kehlkopfdeckel und Kehlkopfeingang mit völligem Verschluß des Kehlkopfes in Höhe der Stimmritzen, Tracheobronchitis, akute passive Hyperämie der Organe, Hirnödem, Lungenödem.

Todesursache: Ersticken bei ödematösem Verschluß des Kehlkopfeinganges (Abb. 4). 


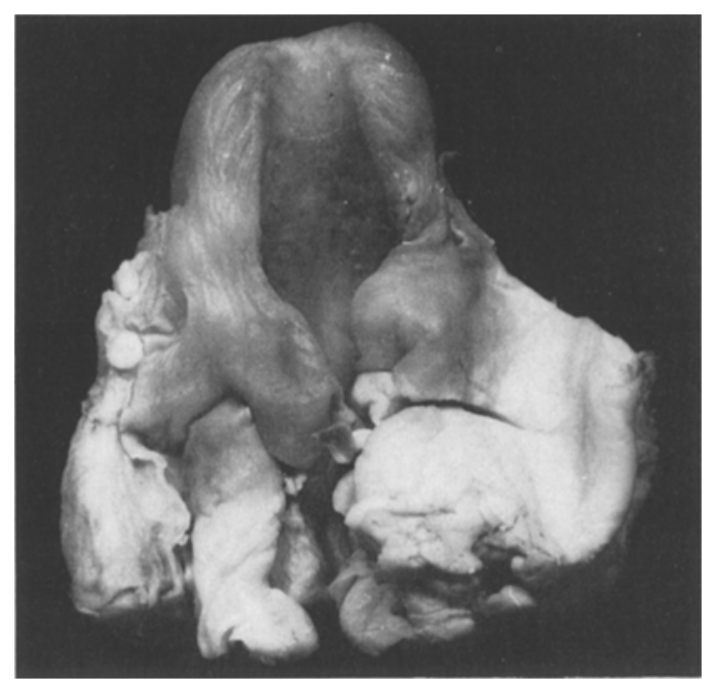

Abb. 4. Ödematöse Schwellung des Kehldeckels und Kehlkopfeinganges.

\section{Fal2 6:}

Ein 32-jähriger Mann klagte nachts bei seiner Vermieterin über Atembeschwerden. Die medikamentöse Behandlung durch den herbeigerufenen Nachtdienstarzt blieb erfolglos, weshalb der Notarzt verständigt wurde. Der Patient begab sich noch selbst zum Notarztwagen. Vom Notarzt wurde zunächst eine Intubation versucht, dann eine Tracheotomie vorgenommen. Trotzdem trat der Tod durch Ersticken ein. Obduktionsbefund (GS 926/74):

Abszeß an der Vorderwand des Keh1decke1s, hochödematöse Schwellung der Keh1deckelschleimhaut mit vollständigem Verschluß des Kehlkopfeinganges, oberflächlicher entzünd1icher Schleimhautdefekt am Keh1deckel zwischen Keh1decke1 und Zungengrund, Lungenödem, entzündliche Milzschwellung, Zustand nach Nottracheotomie mit Blutung in das umgebende Gewebe.

Todesursache: Ersticken bei ödematösem Verschluß des Kehlkopfeinganges.

\section{GESCHWULSTBILDUNGEN}

Ma1igne und benigne Geschwịiste im Kehlkopfbereich sind noch seltener Ursache eines plötz1ichen Todes als die Zysten und Zelen des Keh1kopfes. Zumeist verursachen sie während ihres Wachstums klinisch manifeste Beschwerden, die zu einer rechtzeitigen Behandlung führen. Gefährlich sind gestielte Geschwülste, die mobilisierbar sind und einen Ventilverschluß des Kehlkopfes hervorrufen können. Aus der rechtsmedizinischen Literatur sei hier auf den Fall von CAROFF hingewiesen. Unter den bösartigen Geschwulstbildungen sind die subglottischen Tumoren, die relativ spät Beschwerden verursachen, am ehesten geeignet, einen p1ötz1ichen Tod herbeizuführen. Insgesamt sind Tumoren jedoch in der Gruppe 

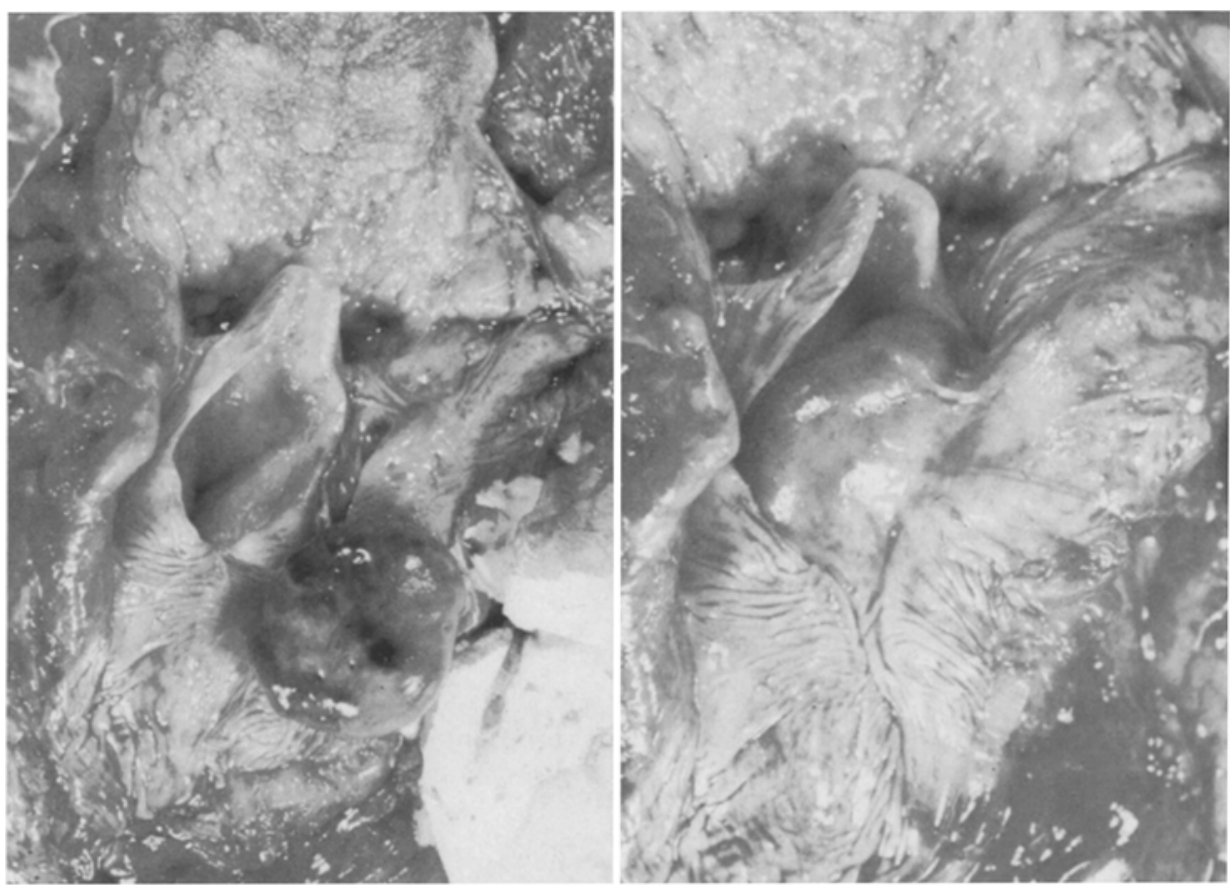

Abb. 5a. Auffindungslage des gestie1ten Oesophgustumors neben dem Kehlkopfeingang

Abb. 5b, Ventilartiger Verschluß des Keh1kopfeinganges durch den Tumor bei seitlicher Lagerung der Halsorgane

der Keh1kopfveränderungen, die einen plötzlichen Tod verursachen können, eine extreme Seltenheit.

Abschließend sei auf einen Fall hingewiesen, bei dem eine gestielte Geschwulst als Nebenbefund festgeste1lt wurde, Dîese Geschwulst hätte, wie sich aus den $A b b$, 5a und $5 b$ ergibt, jederzeit den Kehlkopf verschließen können.

\section{Eal2 7;}

Ein 67-jähriger Mann verstarb nach einem Verkehrsunfa11, bei dem er ausgedehnte Hirnkontusionen erlitten hatte, an einer zentralen Lähmung.

Obduktionsbefund (L 341/67, Freiburg im Breisgau) ${ }^{1}$

Neben den traumatischen Veränderungen fand sich eine kastaniengroße, gestielte Geschwulst der Oesophagusschleimhaut in Kehlkopfnähe, die bei bestimmter Lagerung den Keh1kopfeingang versch1ießen konnte.

1 Für die Überlassung des Sektionsprotokolls danken wir Herrn Prof. Dr. B. FORSTER, Freiburg/Brsg. 


\section{DISKUSSION}

Den Keh1kopferkrankungen als Ursache eines p1ötzlichen Todes kommt in der rechtsmedizinischen Praxis eine relativ geringe Bedeutung zu. Die Todesursache bei natürlichen Erkrankungen ist in aller Regel ein Ersticken bei Keh1kopfverschluß im Gegensatz zu den Reflexmechanismen, die beim Bolustod und bei operativen Eingriffen im Kehlkopfbereich verantwortlich gemacht werden. Die ödematösen Schleimhautschwellungen aus verschiedener Ursache sind häufiger Ursache eines Verschlusses als Fehlbildungen und Tumoren.

Schon bei der makroskopischen Beurteilung bietet sich bei den Kehlkopfödemen eine besondere Schwierigkeit: Der Befund der ödematösen Schwellung der Schleimhäute kann durch postmortale Austrocknung völlig verwischt werden. Zumindest bei den allergischen und angioneurotischen ödemen ist dann auch ein histologischer Nachweis nicht mehr möglich, da entzündliche zelluläre Reaktionen fehlen. Einè starke Faltenbildung der Schleimhäute am Keh1kopfeingang sollte deshalb für den Obduzenten immer Anlaß sein, ẗberlegungen in Richtung auf ein KehIkopfödem anzustellen.

Bemerkenswert erscheint uns in den selbstbeobachteten Fällen, daß punktförmige Blutaustritte in die Bindehäute und unter die serösen Häute, die früher als klassische Erstickungszeichen angesehen wurden; selten zu beobachten sind. Bei einer hochgradigen Einengung ohne Nachweis eines völligen Kehlkopfverschlusses spricht deshalb das Fehlen solcher Blutungen nicht gegen einen Erstickungstod. Auffälig ist auch die Diskrepanz zwischen den anamnestisch bekannt gewordenen Beschwerden und den morphologischen Befunden. Zumindest bei den Larynxzysten oder sich langsam entwickelnden Entzündungen würde man erwarten, daß die Beschwerden den Patienten in ärztliche Behandlung führen müßten. Demgegeniiber ist die Indolenz mancher Personen jedoch nicht zu unterschätzen.

Bei sofortigem Erkennen des plötzlichen oder langsamen Kehlkopfverschlusses bietet aus ärztlicher Sicht die Notfalltracheotomie mit anschließenden Reanimationsmaßnahmen die beste Überlebenschance. Fall 6 zeigt jedoch, daß auch eine Nottracheotomie nicht immer lebensrettend ist. Dies ist bei der Beurteilung des strafrechtlichen Vorwurfes einer Tötung durch Unterlassung zu berïcksichtigen.

Für die forensische Begutachtung ergeben sich im Zusammenhang mit den Larynxzysten weitere Probleme. Die Abgrenzung, ob es sich um eine angeborene Zyste oder eine Retentionszyste aus einem verlegten Ausführungsgang einer Sch1eimdrüse handelt, ist histologisch nicht immer eindeutig abgrenzbar (Fa11 1). Wie oben erwähnt, haben WIGGER und TANG bei einem Säug1ing eine Larynxzyste beobachtet, die als Folge einer. Intubation angesehen wurde. Bei der Vielzahl der heute durchgeführten, teilweise auch länger dauernden Intubationen wäre somit ein 
Kausalzusammenhang, z.B. mit einem Unfallgeschehen, welches die Intubation erforderlich machte, und einer Larynxzyste zu diskutieren.

Letztlich sei noch ein kuratives Problem angeschnitten. Bei Quincke-ödemen sollte alles versucht werden, um ein Allergen nachzuweisen. Nachdem aus der Veröffentlichung von PICK hervorgeht, daß congenitale Störungen des Histaminstoffwechsels familiär gehäuften Quincke-ödemen zugrunde liegen können, wäre aus präventiven Gründen wünschenswert, in den betroffenen Familien aufklärend zu wirken. Leider findet sich im rechtsmedizinischen Alltag nach Abklärung der forensischen Fragestellung keine Gelegenheit zur Wahrnehmung kurativer Maßnahmen.

\section{LITERATUR}

BOSTRöM, K.: Acute Epiglottitis as Cause of Sudden Death. Dtsch. Z. ges. gericht1. Med. 61, 53-58 (1967)

CAROFF, J.: Mort Subite et Fumeur Linguale. Ann. Méd. lég. 45, 527-531 (1965)

DÖRING, L.: Obstruierende Larynxzyste bei einem Neugeborenen. Mschr. Kinderheilk. 121, 684-685 (1973)

KELLER, W., WISKOTT, A.: Lehrbuch der Kinderheilkunde. S. 409, Stuttgart: Thieme-Verlag 1961

KöHN, H.: Kehlkopf und Luftröhre. In: Doerr, Seifert, Üh1inger, Spezielle pathologische Anatomie. Berlin-Heiderlberg-New York: Springer 1969

MATZKER, J.: Über große Zysten und Zelen des Larynxeingangs und seiner Umgebung.

Z. Laryng. Rhinol. 36, 318 (1957)

PICK, J.F.: Sudden Death due to Angioneurotic Oedema. Med. Sci. Law 3, 88-99 (1963)

WIGGER, H.J., TANG, P.: Fata1 Laryngeal Obstruktion by Jatrogenic Subglottic Cyst. J. Pediat. 72, 815-820 (1968)

Drs, med. W. EISENMENGER, G. BECKMANN

H. -D. TRÖGER

Inst. für Rechtsmedizin der Universität

D-8000 München 2, Frauenlobstr. 7 a

Bundesrepublik Deutschland 